\title{
Discriminación laboral de las mujeres en las Pymes del Sur de Sonora
}

\section{Labor discrimination of women in SMEs in Southern Sonora}

\author{
VALDEZ-PINEDA, Dina Ivonne*† \& OCHOA-JAIME, Blanca Rosa \\ Instituto Tecnológico de Sonora. Dirección de Ciencias Económico Administrativa. Calle 5 de Febrero 818, Centro, Urb.
} No. 1, 85000 Cd Obregón, Son.

ID $1^{\text {er }}$ Autor: Dina Ivonne, Valdez-Pineda / ORC ID: 0000-0001 8660-1787, Researcher ID Thomson: S6895-2018, CVU CONACYT ID: 282771

ID $1^{\text {er }}$ Coautor: Blanca Rosa, Ochoa-Jaime / ORC ID: 0000-0002-3387-632X, Researcher ID Thomson: S-6886-2018, CVU CONACYT ID: 688073

DOI: $10.35429 / J L A .2019 .7 .3 .1 .7$

Recibido 11 de Enero, 2019; Aceptado 25 de Marzo, 2019

\section{Resumen}

La discriminación laboral y la que aqueja a las mujeres en particular, frena el desarrollo de la sociedad y lo hace de manera grave en un país como el nuestro. Tomando en cuenta lo anterior se plantea el siguiente interrogante ¿Cuál es la principal causa de discriminación laboral de las mujeres en las PYMES del Sur de Sonora? El objetivo es identificar las causas que influyen en la discriminación laboral de mujeres en las PYMES del Sur de Sonora. El método utilizado fue descriptivo de tipo cuantitativo, con una muestra de 250 mujeres trabajadoras del sur de Sonora. Los resultados obtenidos fueron que la discriminación laboral para mujeres con hijos, argumentando que faltan al trabajo por atender a los hijos, por políticas de la empresa y por ideas erróneas que tienen los jefes a cargo respecto a las mujeres con hijos. Concluyendo que la discriminación laboral de las mujeres es una situación que debe ser motivo de preocupación debido a las graves consecuencias que ocasionan. Él no poder contribuir en el sustento de la familia, la formación personal de los hijos y que por ende pueden ser causas de desvíos negativos de personalidad con repercusión a la sociedad.

Discriminación, Discriminación Laboral, PYMES

\begin{abstract}
Labor discrimination and that which afflicts women in particular, slows the development of society and does so in a serious way in a country like ours. Taking into account the above, the following question arises: What is the main cause of employment discrimination for women in SMEs in Southern Sonora? The objective is to identify the causes that influence the discrimination of women in SMEs in the South of Sonora. The method used was descriptive of quantitative type, with a sample of 250 working women from the south of Sonora. The results obtained were that labor discrimination for women with children, arguing that they miss work because of caring for their children, because of company policies and because of mistaken ideas held by the chiefs in charge of women with children. Concluding that the employment discrimination of women is a situation that should be cause for concern due to the serious consequences they cause. He can not contribute to the sustenance of the family, the personal formation of the children and that therefore can be causes of negative deviations of personality with repercussion to the society.
\end{abstract}

Discrimination, Labor Discrimination, PYMES

Citación: VALDEZ-PINEDA, Dina Ivonne \& OCHOA-JAIME, Blanca Rosa. Discriminación laboral de las mujeres en las Pymes del Sur de Sonora. Revista de Aplicaciones del Derecho. 2019. 3-7: 1-7.

\footnotetext{
*Correspondencia al Autor (Correo Electrónico: dvaldez@itson.edu.mx)

$\dagger$ Investigador contribuyendo como primer autor.
} 


\section{Introducción}

Hoy en día las mujeres de todo el mundo se mantienen en una lucha constante buscando obtener mayores oportunidades e igualdad dentro de la cultura machista y prácticas sociales discriminatorias que existen principalmente en nuestro país. A través de este esfuerzo las mujeres han demostrado que son capaces de llevar a cabo muchas de las actividades que realizan los hombres, y con ello se ha logrado avanzar pero no de forma significativa.

El Consejo Nacional para Prevenir la Discriminación en México, (CONAPRED, 2018) ha recibido más mil 726 quejas presentadas por mujeres desde 2011 hasta la fecha, de las cuales $73 \%$ están relacionadas con actos de discriminación en el mundo laboral.

La Encuesta Nacional sobre Discriminación (ENADIS, 2010) realizada por el CONAPRED, muestra en sus resultados que el $50 \%$ de las mujeres concuerdan que en México no son respetados sus derechos, lo cual es considerado como un tipo de violencia de género, determinado por la discriminación y que les afecta directamente en el ejercicio y goce de sus derechos.

El Instituto Nacional de las Mujeres [INMUJERES], la Secretaria de Trabajo y Prevención Social [STPS] y el CONAPRED (2015), en conjunto impulsan actualmente la adopción de la Norma Mexicana en NMX-R025-SCFI-2015 en Igualdad Laboral y No Discriminación en Inclusión Laboral, la cual prevé que los hombres y las mujeres gocen de los mismos derechos, buscando con ello erradicar la discriminación y ofrecer a las mujeres trabajos bien remunerados en igualdad de oportunidades. Se busca a través de ello que las empresas públicas, privadas y sociales en México, adopten voluntariamente prácticas en materia de igualdad laboral y no discriminación, que puedan favorecer al igual a hombres y mujeres, sin importar sector ni actividad, y se logre incorporar la perspectiva de género y no discriminación en diversos procesos.

En base a los puntos antes mencionados se puede observar la gran afectación que existe actualmente a las mujeres en el ámbito laboral, ya que en algunas empresas son discriminadas por el hecho de ser mujer.
Por lo que se consideró pertinente realizar esta investigación para determinar el grado de discriminación laboral para las mujeres en Pymes del Sur de Sonora.

\section{Justificación}

Los derechos de los individuos en la sociedad actual exigen que todas las personas sean tratadas con igualdad y sin discriminación. Este principio de igualdad ha generado hasta nuestros días una lucha incansable por lograr los mismos derechos humanos entre hombres y mujeres y es la intención que motiva la realización de esta investigación. La discriminación de las mujeres trabajadoras en México es preocupante puesto que un mayor número de mujeres hoy en día se desempeñan laboralmente pretendiendo aportar parte al sustento familiar que les permita mejorar la calidad de vida de sus familias.

De los argumentos antes mencionados se desprende la iniciativa de la realización del presente estudio de investigación, y la importancia del mismo, ya que el tema de discriminación laboral de la mujer es hoy en día es uno de los más preocupantes por el papel tan importe de la mujer en este entorno y es necesario conocer a fondo todas las causas que originan esta situación y que a su vez sirva para tomar medidas que contribuyan a combatir este tipo de acciones en el ámbito laboral.

\section{Problema}

La discriminación laboral hacia mujeres es problema social de mucho tiempo atrás, desde la antigua sociedad las mujeres fueron consideradas como el sexo inferior, eran tratadas con crueldad y se consideraban sin ningún derecho, se decía que no eran dueñas de su propia vida y además eran incapaces de tomar decisiones propias.

Con este estudio se busca identificar el grado de discriminación laboral que sufren las mujeres en la PYMES del Sur de sonora, por lo tanto, esta investigación está enfocada a detectar las posibles causas que originan esta situación. De lo anterior surge la interrogante: ¿Cuáles son las principales causas de discriminación laboral de mujeres en las PYMES del Sur de Sonora? 


\section{Objetivo}

Identificar las causas que influyen en la discriminación laboral de mujeres en las PYMES del Sur de Sonora.

\section{Marco teórico}

"La discriminación es una práctica cotidiana que consiste en dar un trato desfavorable o de desprecio inmerecido a determinada persona o grupo, que a veces no percibimos, pero que en algún momento la hemos causado o recibido". (CONAPRED, 2018). Sus efectos se consideran negativos y se relacionan con la pérdida de derechos y la desigualdad, incluso está considerada como una forma de violencia pasiva.

Por su parte la Convención para la Eliminación de Todas las Formas de Discriminación contra la Mujer, [CETFDMC], en su artículo 1, la define como "toda distinción, exclusión o restricción basada en el sexo, que tenga por objeto o resultado anular o menoscabar el reconocimiento, goce o ejercicio de los derechos de la mujer, independiente de su estado civil, sobre la base de la igualdad del hombre y la mujer, de los derechos humanos y las libertades fundamentales".

La Convención tiene como objetivo eliminar efectivamente todas las formas de discriminación contra la mujer, obligando a los estados a reformar las leyes con tal fin y discutir sobre la discriminación en el mundo.

La discriminación es considerada un fenómeno social que daña la dignidad, los derechos humanos y las libertades fundamentales de las personas o grupos, a través de tratos de inferioridad, basados en criterios como la raza, el origen étnico o nacional, opiniones, entre otros aspectos (CNDH, 2012).

El trabajo es un derecho que toda persona tiene, y por tanto básico para su supervivencia, aunque en algunos casos las empresas siguen discriminando a la mujer pues erróneamente creen que no tienen la habilidad para llevar a cabo el trabajo que se le pudiera encomendar.
La no discriminación se encuentra regulada en la Carta Magna y en diferentes tratados e instrumentos que luchan para combatir la discriminación contra la mujer, La Constitución Política Mexicana en su artículo 1 establece la "prohibición de todo tipo de discriminación motivada por origen étnico o nacional, el género, la edad, las discapacidades, la condición social, las condiciones de salud, la religión, las opiniones, las preferencias, el estado civil o cualquier otra que atente contra la dignidad humana y tenga por objeto anular o menoscabar los derechos y libertades de las personas" (DIPUTADOS, 2016).

El artículo Cuarto establece que el hombre y la mujer son iguales ante la ley y por lo tanto, el Estado está obligado a proteger la organización y desarrollo de la familia (DIPUTADOS, 2016). En ese mismo tenor, el artículo 123 en su apartado A, fracciones V y VII "protege la maternidad de la mujer, e indica que toda persona tiene derecho al trabajo digno y socialmente útil; para ello se promoverá la creación de empleos y la organización social de trabajo, conforme la ley" (DIPUTADOS, 2016).

La Ley Federal del Trabajo, en su artículo 2, define "al trabajo digno como aquel en el que se respeta plenamente la dignidad humana del trabajador; asimismo, prohíbe la discriminación en el ámbito laboral por origen étnico o nacional, género, edad, discapacidad, condición social, condiciones de salud, entre otros aspectos. De igual forma, asegura que se tiene acceso a la seguridad social y a un salario remunerado; a capacitaciones continuas para el incremento de la productividad con beneficios compartidos, y a condiciones óptimas de seguridad e higiene para prevenir riesgos de trabajo".

Las regulaciones no han sido suficiente para erradicar las prácticas discriminatorias en el ámbito laboral, pues en algunos casos el salario no se paga conforme la ley, siendo la mujer la que recibe un mayor perjuicio por su condición de género. 
Igualmente, y debido a estereotipos de género, no se confía en su capacidad en determinados sectores o trabajos en particular; en materia laboral, el artículo 1, inciso a del Convenio sobre la Discriminación (empleo y ocupación) de la Organización Internacional del Trabajo (OIT, 1958) señala que se considera discriminación a cualquier distinción, exclusión o preferencia basada en motivos de raza, color, sexo, religión, opinión política, ascendencia nacional $\mathrm{u}$ origen social que tenga por efecto anular o alterar la igualdad de oportunidades o de trato en el empleo y la ocupación.

La discriminación hace referencia a la alteración o modificación de la igualdad entre las personas, ya sea por reducción, exclusión o restricción; cuando se alternan las condiciones de igualdad en el trabajo, se habla de discriminación laboral, principalmente cuando no hay oportunidades en el trato, o bien diferencia en las prestaciones o en el pago, teniendo como origen el sexo y el género, así como cualquier otro atributo que modifique el esquema de igualdad (Kureczyn V., 2004).

La discriminación se clasifica en dos formas: Es considerada directa cuando existen reglas o prácticas que abiertamente estigmatizan o excluyen a las personas lesionando sus derechos; en el ámbito laboral se presenta cuando en un determinado trabajo, la oferta sólo va dirigida a un género en específico, por lo tanto, la parte opuesta no tiene la posibilidad de postularse. Ejemplo de esto, cuando una compañía necesita electricista, de sexo masculino (INMUJERES, ¡Vive sin discriminar!, Discriminación directa e indirecta, S.F.).

En cambio la llamada discriminación indirecta resulta de la vigencia de reglas o prácticas que aparentemente son neutrales, pero cuyos efectos excluyen derechos fundamentales, como en el caso de una empresa que requiere a una persona con disponibilidad de horario; lo que hace pensar que en estos casos el hombre tiene la ventaja, ya que si bien no ponen género, la mujer tradicional por el hecho de ser madre y esposa no cuenta con el requisito establecido porque no tendrá esa disposición de horario solicitada (INMUJERES, ¡Vive sin discriminar!, Discriminación directa e indirecta, S.F.).
La discriminación se genera por el género, como cuando la mujer sale perjudicada, dado que en ocasiones se le vulnera por sus capacidades físicas en el ámbito laboral y en determinados puestos, pues se considera menos apta para llevarlo a cabo. O bien, puede existir la discriminación por la edad, considerando que en ocasiones no se les da trabajo a las personas mayores de 50 años, porque no se confía en su capacidad física para ejercer determinados trabajos, aun cumpliendo con los requisitos necesarios.

El trabajo es un derecho que toda persona tiene, y por tanto básico para su supervivencia, aunque en algunos casos las empresas siguen discriminando a la mujer pues erróneamente creen que no tienen la habilidad para llevar a cabo el trabajo que se le pudiera encomendar.

\section{Metodología de Investigación}

La investigación posee un alcance descriptivo, cuantitativa con un diseño no experimental. La información fue recolectada, a través de una encuesta personal, en un solo momento, durante los meses de agosto a diciembre del 2018.

Los sujetos de estudio fueron las empleadas de PYMES del Sur de Sonora, se seleccionó una muestra no probabilística por conveniencia de 250 Trabajadoras en PYMES y que hayan trabajado anteriormente (Se determinó la muestra por conveniencia por la poca participación y tiempos de las empleadas para contestar la encuesta).

El instrumento aplicado en el presente estudio de investigación es un cuestionario estructurado y validado de acuerdo a la problemática planteada, integrado por cinco preguntas cerradas, de las cuales dos eran de respuesta dicotómica en las que se preguntaba si le han negado la oportunidad de trabajar por ser mujer y si la habían discriminado por estar embarazada; y tres de opción múltiple en las que se cuestionaba las causas de la negación del trabajo, causas de la discriminación y la etapa más difícil para conseguir trabajo por ser mujer, para lo cual se utilizó una gama de respuestas relacionadas con el tema investigación tratando de identificar causas que originan discriminación laboral de las mujeres en el sur de Sonora. 


\section{Procedimiento}

Se realizó la elección del problema que se desea investigar, se definieron las variables considerando los factores que inciden en la discriminación laboral y género. Se determinó la población de las mujeres que trabajan en PYMES en el sur de Sonora. Se llevó acabo la aplicación del instrumento en las PYMES seleccionadas en la muestra.

Se capturaron y analizaron los datos recabados mediante el sistema Statistical Package for the Social Sciences (SPSS), utilizando una prueba-T para evaluar los 7 ítems. Para valorar las distintas variables con una escala tipo Likert.

\section{Resultados}

Se encuestaron a 250 mujeres trabajadoras en PYMES del Sur de Sonora, con una edad promedio de 24 años. En este estudio se presentarán algunos de los aspectos que fueron evaluados en el instrumento y que se consideran como más relevantes para determinar la causa de discriminación laboral.

En el rubro de que si alguna vez le han negado la oportunidad del trabajo por ser mujer se encontró que el $68 \%$ de las mujeres encuestadas contestaron que si le han negado él empleo por ser mujer, mientras que un $32 \%$ contesto que no (ver Gráfico 1).

\section{¿Alguna vez le han negado una oportunidad de empleo, por ser mujer?}

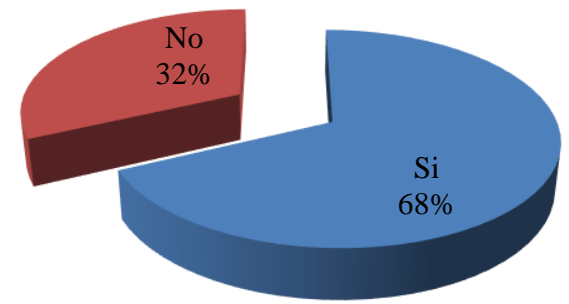

Gráfico 1 Negación de empleo por ser mujer Fuente: elaboración propia

\section{Causa principal de la negación del trabajo}

El $49 \%$ de las mujeres trabajadoras que se les negó el trabajo contestaron que por tener hijos, el $36 \%$ por estar casada y un $15 \%$ por el solo hecho de ser mujer (ver Gráfico 2).

\section{¿Cuál ha sido la causa principal en la negación de un empleo?}
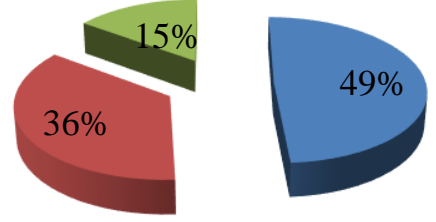

por tener
hijos
por estar
casada

Gráfico 2 Causas de negación de empleo Fuente: elaboración propia

\section{Causa principal de la discriminación laboral de la mujer}

Del total de mujeres trabajadoras el $45 \%$ cree que la causa principal de la discriminación laboral es la posible tendencia de faltar al trabajo por atender a los hijos. Un $20 \%$ considera que por el poco rendimiento que tendrían por tener dos responsabilidades, otro $20 \%$ comenta que por políticas de la empresa, un $10 \%$ opina que por las ideas erróneas que tienen los jefes sobre el rendimiento de las mujeres y un $5 \%$ que por posibles embarazos (ver Gráfico 3).

\section{¿Cuál ceee que sea la causa principal por la discriminación laboral de la mujer?}

- posiblies tendencias de faltar al trabajo por atender a los hijos - Poco rendimiento laboral por tener dos responsabilidades - por politicas de la empresa

- Algunos jefes tienen ideas erroneas sobre las mujeres

- Posibilidades de embarazos

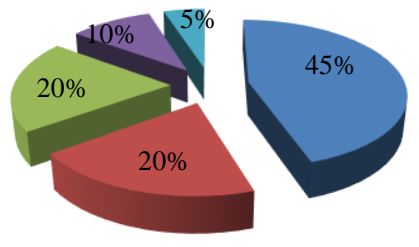

Gráfico 3 Causas de discriminación laboral Fuente: elaboración propia

\section{Etapa más difícil para la mujer para conseguir trabajo}

Del $100 \%$ de las trabajadoras encuestadas menciona el $43 \%$ que la etapa más difícil de la mujer para conseguir trabajo es la etapa de adulta joven de 25 a 35 años de edad e, le sigue la etapa de Jóvenes de 18 a 25 años con un $32 \%$ y un $25 \%$ en la etapa de adulta de 35 a 50 años. Los porcentajes más alto se deben que son en la edad de procrear hijos y que por eso es más difícil su contratación. 
Son en estas etapas donde las empresas no quieren contratar mujeres en determinadas áreas con la justificación de que el costo es mayor que el contratar a un hombre, debido a que genera gastos cuando sale embarazada, lo que lleva a pedir permisos para la lactancia y cuidados maternos al bebe (ver Gráfico 4).

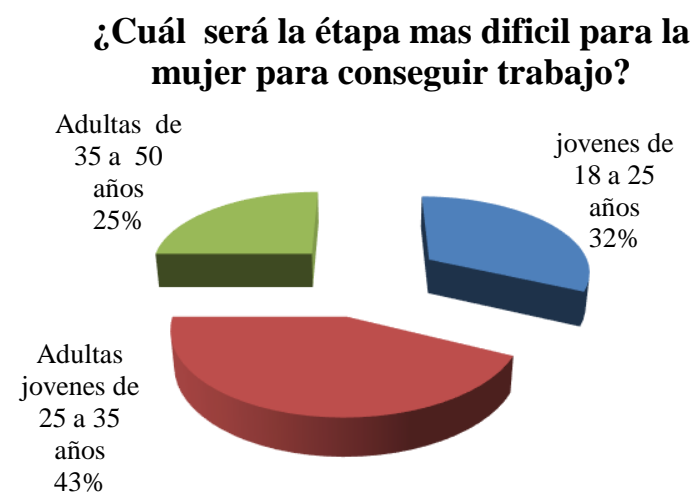

Gráfico 4 Etapas difíciles para conseguir empleo Fuente: elaboración propia

\section{Discriminación laboral por estar embarazada}

El $90 \%$ dice que no han sido discriminadas laboralmente por estar embarazadas y el $10 \%$ contesto que si lo han sido. Aunque aparentemente se están cambiando las perspectivas de trabajo hacia la mujer aún tiene que elegir entre ser madre o trabajadora. Y no es que a ellas les afecte el embarazo, sino que "laboralmente" su rendimiento no es suficiente para continuar con su empleo.

Dentro de una empresa el hecho de no contratar a una mujer por su estado o despedirla por lo mismo, es causa suficiente de ilegalidad (ver Gráfico 5).

\section{¿Alguna vez te han discriminado laboralmente por estar embarazada?}

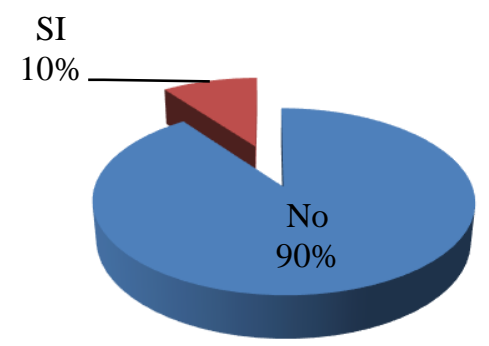

Gráfico 5 Discriminación por embarazo Fuente: elaboración propia

\section{Conclusiones}

La igualdad de oportunidades es un derecho universal reconocido por todas las sociedades democráticas, relacionado con la dignidad y libertad humanas. No es tan solo una cuestión que afecte a las mujeres, sino a la sociedad en general, tanto hombres como mujeres. La igualdad de oportunidades va unida a la no discriminación por razón de sexo.

En la sociedad mexicana, las mujeres siguen siendo objeto de discriminación, lo que les impiden participar en igualdad de condiciones con el hombre y lograr el desarrollo pleno y completo de sus capacidades en su bienestar personal, familiar y social. Ambos géneros constituyen el soporte fundamental de la familia y contribuyen al bienestar de la misma con actividades propias de cada uno, para lograr el desarrollo de la sociedad.

En este estudio la discriminación laboral a mujeres con hijos fue la causa principal por la que no la contrataran en la PYMES del Sur de Sonora. Una situación que debe ser motivo de preocupación debido a las graves consecuencias que pueden traer consigo él no poder contribuir en el sustento de la familia y que a su vez afecta a la formación personal de los hijos y que por ende pueden ser causas de desvíos negativos de personalidad con repercusión a la sociedad.

Otra consecuencia que también ocurre en el ámbito laboral es la discriminación por razones de embarazo. Éste se produce cuando un empleado trata desfavorablemente a una mujer empleada o solicitante de un puesto de trabajo por estar embarazada, parto o trastornos médicos relacionados con el embarazo o la maternidad; o bien, cuando se discrimina y excluye a las mujeres de puestos de trabajo determinados, debido a que pueden quedar embarazadas y ante la idea de que no podrán ejercer su trabajo como debe ser.

Según Antonia May de la Cruz y José Antonio Morales Notario (2018). La discriminación laboral por género aún es palpable en nuestra sociedad. Sin embargo, el problema irá desapareciendo debido a que las empresas cada vez juegan un papel fundamental e indispensable en este tema, además de que continuamente muestran preocupación, por la presión internacional y la globalización e internacionalización.

VALDEZ-PINEDA, Dina Ivonne \& OCHOA-JAIME, Blanca Rosa. Discriminación laboral de las mujeres en las Pymes del Sur de Sonora. Revista de Aplicaciones del Derecho. 2019 
Atendiendo las necesidades y motivando a los trabajadoras/es sin importar el género, se lograrán los objetivos y metas que se propongan, los cuales pueden ser cumplidos en un ambiente de igualdad y equidad. La visión de las empresas debe estar enfocada en la inclusión de la mujer al mundo laboral, sin importar su género, pero tomando en consideración sus funciones.

$\mathrm{Si}$ se toma conciencia de que la discriminación daña a la sociedad y se toman las medidas adecuadas para prevenir los casos que surgen de las relaciones laborales, entonces se logrará beneficiar a las empresas y a los trabajadores. Un ambiente menos machista, más equilibrado entre géneros y con mejores condiciones en las empresas de nuestro país logrará la equidad laboral.

\section{Referencias}

Consejo Nacional para Prevenir la Discriminación en México [CONAPRED 2018]. Secretaria de Gobernación. Recuperado de: http://www.conapred.org.mx/

CNDH. (2012). La discriminación y el derecho a la no discriminación. México: Comisión Nacional de Derechos Humanos.

DIPUTADOS. (2016). Constitución Política de los Estados Unidos Mexicanos. Obtenido de Cámara de Diputados del H. Congreso de la Unión, Secretaría General: http://www.diputados.gob.mx/LeyesBiblio/pdf/ 1_240217.pdf

Encuesta Nacional sobre Discriminación en México [ENADIS, 2010]. Consejo Nacional para Prevenir la Discriminación en México. Recuperado de: http://www.conapred.org.mx/userfiles/files/Ena dis-2010-RG-Accss-002.pdf.

INMUJERES. (S.F.). ¡Vive sin discriminar!, Discriminación directa e indirecta. Obtenido de Instituto Nacional de las Mujeres: http://puntogenero.inmujeres.gob.mx/madig/dis criminacion/seccion2.html.

May, Morales (2017). La discriminación laboral de la mujer en México. Recuperado de: file:///C:/Users/acer/Downloads/2243-9820-1PB.pdf
Kurezyn V. P. (2004). Acoso sexual y Discriminación. México: UNAM.

OIT. (1958). Convenio sobre la discriminación (empleo y ocupación), número 111. Convenio relativo a la discriminación en materia de empleo y ocupación. Ginebra: OIT. 